\title{
Theory of Probabilistic Connectedness
}

\author{
Yu-Lin Chou*
}

\begin{abstract}
We introduce and study a notion of probabilistic connectedness, which we term proconnectedness, defined in terms of partitions of a probability space into two nonempty disjoint independent events. Both proconnectedness and disproconnectedness are shown to be invariants (in a suitable sense) under isomorphic random elements. We show that a substantial part of the fundamental theory of topological connectedness admits a natural counterpart in the present theory of proconnectedness. Some applications and connections regarding limit theorems, cardinality equality of measurability structures, atomic distributions, and singular distributions are discussed.
\end{abstract}

Keywords: connectedness; independence; isomorphisms; proconnectedness; probabilistic invariants; singular distributions

MSC 2020: 60A10; 60A99; 60H99; 60E05

\section{Introduction}

The notion of independence, in particular that of independence of two events of a probability space, is arguably a characteristic that differentiates probability theory from general measure theory. Independence not only satisfactorily captures the natural notion of independence associated with empirical experiments, but is also of intrinsic interest; the literature of limit theorems in probability theory serves as a significant example.

In the present work, independence plays a role even more abstract. We introduce and study a new legitimate notion of connectedness for probability spaces, which is different than the usual notion of topological connectedness precisely in replacing open sets with independent measurable sets. It turns out that both probabilistic connectedness and probabilistic disconnectedness are invariants under bimeasurable bijective random

*Yu-Lin Chou, Institute of Statistics, National Tsing Hua University, Hsinchu 30013, Taiwan, R.O.C.; Email: y.1.chou@gapp.nthu.edu.tw 
elements in a natural sense; and, unexpectedly, many fundamental results in the theory of topological connectedness are preserved in that of our probabilistic connectedness.

We also record as applications some interesting results as suggested by our theory of probabilistic connectedness.

\section{Results}

We begin with defining the new notion of probabilistc connectedness:

Definition 1. Let $(\Omega, \mathscr{F}, \mathbb{P})$ be a probability space. Then $\Omega$ is said to be disproconnected- $\mathbb{P}$ if and only if there are nonempty disjoint $A_{1}, A_{2} \in \mathscr{F}$ such that $A_{1} \cup A_{2}=\Omega$ and $A_{1}, A_{2}$ are independent- $\mathbb{P}$.

The probability space $\Omega$ is said to be proconnected- $\mathbb{P}$ if and only if $\Omega$ is not disproconnected- $\mathbb{P}$.

Thus the role of independent events in the definition of proconnectedness is that of open sets in the definition of connectedness. When the underlying probability measure is offstage, the terms "proconnected" and "disproconnected" are understood with respect to Definition 1.

Proconnectedness is not a vacuous notion:

Example 1. If $\Omega$ is a singleton with $\mathbb{P}$ denoting the Dirac measure defined on the trivial sigma-algebra $\{\varnothing, \Omega\}$, then $\Omega$ is proconnected- $\mathbb{P}$.

If $\Omega$ is a set with cardinality $=2$, and if $\mathbb{P}$ is the Dirac measure on $2^{\Omega}$ concentrated at an element of $\Omega$, then $\Omega$ is disproconnected- $\mathbb{P}$. Indeed, if $\Omega=\{x, y\}$, and if $\mathbb{P}$ is concentrated on $\{x\}$, then

$$
\mathbb{P}(\{x\} \cap\{y\})=0=\mathbb{P}(\{x\}) \mathbb{P}(\{y\}) .
$$

A slightly wilder and informative example of a disproconnected space may be considered as well. For instance, if $\mathbb{R}$ is taken as a Gaussian probability space with $\mathbb{P}$ denoting the given Gaussian distribution, then, since $\mathbb{P}$ is Lebesgue-absolutely continuous, the partition $\{\mathbb{Q}, \mathbb{R} \backslash \mathbb{Q}\}$ makes $\mathbb{R}$ disproconnected-P.

More interesting examples will come into play later on.

Conceivably, since proconnectedness depends on probability measures, it has little to do with connectedness. For concreteness, we examine the following

Example 2. (i) Connectedness sometimes coincides with proconnectedness: If $\Omega$ is a singleton, then $\Omega$ is connected (with respect to the trivial topology, certainly); but Example 1 shows that $\Omega$ is also proconnected when equipped with the Dirac measure. 
(ii) Disconnectedness sometimes coincides with disproconnectedness: If $\Omega$ is a set with cardinality $=2$, then $\Omega$ is disconnected with respect to the discrete topology of $\Omega$; but Example 1 shows that $\Omega$ is also disproconnected with respect to a suitable Dirac measure.

(iii) There is some connected space that is disproconnected: If $\Omega$ is a Sierpiński space, then $\Omega$ is trivially connected. But since $\Omega$ by definition has cardinality $=2$, the same choice of Dirac measure in (ii) proves the claim.

(iv) There is some proconnected space that is disconnected: If $\Omega$ is a discrete topological space with cardinality $=2$, then $\Omega$ is disconnected. But, if $\mathbb{P}_{1}$ is the Dirac measure concentrated at an element of $\Omega$, and if $\mathbb{P}_{2}$ is the Dirac measure concentrated at the other element of $\Omega$, then $\Omega$ is proconnected $-\left(\frac{1}{2} \mathbb{P}_{1}+\frac{1}{2} \mathbb{P}_{2}\right)$; the only partition of $\Omega$ into two nonempty measurable sets does not have independent elements.

The induced probability measure of a given probability measure $\mathbb{P}$ by a measurable map $X$ will be denoted by $\mathbb{P}_{X}$. For every map $f$, we denote by $f^{-1}$ ) the preimage map and by $f^{1)}$ the image map corresponding to $f$. A sigma-algebra $\mathscr{F}$ relativized to an element $A \in \mathscr{F}$ will be denoted by $\mathscr{F}\rceil_{A}$; and, if $\mathbb{P}$ is a probability measure on $\mathscr{F}$, then $\mathbb{P}\rceil_{A}(\underline{A}):=\mathbb{P}(A \cap \underline{A})$ for all $\underline{A} \in \mathscr{F}$. (The notation $\rceil$ is adapted from Federer's notation in Federer [1], perhaps the earliest one in the related literature.) A measurable map (i.e. a random element [of the codomain]) preserves proconnectedness in the following (natural) sense:

Theorem 1. Let $(\Omega, \mathscr{F}, \mathbb{P})$ be a probability space that is proconnected- $\mathbb{P}$; let $(\underline{\Omega}, \underline{\mathscr{F}})$ be a measurable space. If $X: \Omega \rightarrow \underline{\Omega}$ is measurable- $(\mathscr{F}, \underline{\mathscr{F}})$, then $\underline{\Omega}$ is proconnected $-\mathbb{P}_{X}$.

Proof. If $\underline{\Omega}$ is disproconnected- $\mathbb{P}_{X}$, then there are some nonempty disjoint $A_{1}, A_{2} \in \underline{\mathscr{F}}$ such that $A_{1} \cup A_{2}=\underline{\Omega}$ and $A_{1}, A_{2}$ are independent- $\mathbb{P}_{X}$. But since

$$
\begin{aligned}
& \mathbb{P}\left(X^{-1)}\left(A_{1}\right) \cap X^{-1)}\left(A_{2}\right)\right) \\
& =\mathbb{P}_{X}\left(A_{1} \cap A_{2}\right) \\
& =\mathbb{P}_{X}\left(A_{1}\right) \mathbb{P}_{X}\left(A_{2}\right) \\
& =\mathbb{P}\left(X^{-1)}\left(A_{1}\right)\right) \mathbb{P}\left(X^{-1)}\left(A_{2}\right)\right),
\end{aligned}
$$

the space $\Omega$ is disproconnected- $\mathbb{P}$.

Throughout, an isomorphism acting between measurable spaces is precisely a bimeasurable bijection. Now proconnectedness is by Theorem 1 an invariant under isomorphisms:

Corollary 1. Every measurable space isomorphic to a given proconnected probability space is proconnected with respect to the induced probability measure. 
Some preliminary characterization for disproconnectedness would be convenient:

Proposition 1. Let $(\Omega, \mathscr{F}, \mathbb{P})$ be a probability space. Then $\Omega$ is disproconnected- $\mathbb{P}$ if and only if there is some nonempty $A \in \mathscr{F}$ such that $A^{c}$ is nonempty and $\mathbb{P}(A)=0$.

Proof. The "if" part follows immediately from the definition of independence.

For the "only if" part, let $\Omega$ be disproconnected- $\mathbb{P}$, so that there are some nonempty disjoint $A_{1}, A_{2} \in \mathscr{F}$ such that $A_{1} \cup A_{2}=\Omega$ and $\mathbb{P}\left(A_{1} \cap A_{2}\right)=\mathbb{P}\left(A_{1}\right) \mathbb{P}\left(A_{2}\right)$. Since $\mathbb{P}\left(A_{1} \cap A_{2}\right)=0$, without loss of generality let $\mathbb{P}\left(A_{1}\right)=0$. If $A:=A_{1}$, then both $A$ and $A^{c}$ are nonempty; this completes the proof.

Now disproconnectedness is also an invariant under isomorphisms:

Theorem 2. Let $(\Omega, \mathscr{F}, \mathbb{P})$ be a probability space that is disproconnected- $\mathbb{P} ;$ let $(\underline{\Omega}, \underline{\mathscr{F}})$ be a measurable space. If $X: \Omega \rightarrow \underline{\Omega}$ is an isomorphism, then $\underline{\Omega}$ is disproconnected- $\mathbb{P}_{X}$.

Proof. There is by Proposition 1 some nonempty $A \in \mathscr{F}$ such that $A^{c}$ is nonempty and $\mathbb{P}(A)=0$. Since $X$ is in particular an injection, we have $\mathbb{P}_{X}\left(X^{1)}(A)\right)=\mathbb{P}(A)$ and $\mathbb{P}_{X}\left(X^{1)}\left(A^{c}\right)\right)=\mathbb{P}\left(A^{c}\right)$; moreover, the sets $X^{1)}(A), X^{1)}\left(A^{c}\right)$ are disjoint. The desired conclusion then follows from the relation $X^{1)}(\Omega)=X^{1)}(A) \cup X^{1)}\left(A^{c}\right)$.

For proconnectedness, we have some characterizations reminiscent of those for connectedness:

Theorem 3. Let $(\Omega, \mathscr{F}, \mathbb{P})$ be a probability space. Then (i) $\Omega$ is proconnected-P if and only if (ii) $\left\{A_{1}, A_{2}\right\} \subset \mathscr{F}$ being a partition of $\Omega$ with $A_{1}, A_{2}$ independent implies $\left\{A_{1}, A_{2}\right\}=\{\varnothing, \Omega\}$, if and only if (iii) there is no surjection $X: \Omega \rightarrow\{0,1\}$ such that $X$ is measurable- $\left(\mathscr{F}, 2^{\{0,1\}}\right)$ and $\mathbb{P}_{X}(\{0\}) \mathbb{P}_{X}(\{1\})=0$.

Proof. Since (ii) is equivalent to the statement that there is no partition of $\Omega$ into two nonempty, disjoint, and independent events, the equivalence between (i) and (ii) follows directly from Definition 1.

To show that (i) implies (iii), let $X: \Omega \rightarrow\{0,1\}$ be a measurable surjection. Then $X^{-1)}(\{0\}) \neq \varnothing, \Omega$. Since $X^{-1)}(\{0\}), X^{-1)}(\{1\})$ are nonempty, disjoint, and measurable, if $\mathbb{P}_{X}(\{0\}) \mathbb{P}_{X}(\{1\})=0$ then $X^{-1)}(\{0\})$ is $\mathbb{P}$-independent of $X^{-1)}(\{1\})$, contradicting (i).

There remains to show that (iii) implies (i). If $\Omega$ is disproconnected- $\mathbb{P}$, then there is by Proposition 1 some nonempty $A \in \mathscr{F}$ such that $A^{c}$ is nonempty and $\mathbb{P}(A)=0$. But then the indicator function $\mathbb{1}_{A}: \Omega \rightarrow\{0,1\}$ of $A$ is a measurable surjection with $\mathbb{P}\left(\mathbb{1}_{A}^{-1)}(\{1\})\right)=0$. This completes the proof. 
Our use of "either $\cdots$ or ..." is simply for the purpose of emphasis, which is understood as the usual logical connector "or". Proconnectedness and disproconnectedness of subsets of a given probability space are defined as follows:

Definition 2. Let $(\Omega, \mathscr{F}, \mathbb{P})$ be a probability space; let $\underline{\Omega} \in \mathscr{F}$ have cardinality $\geq 2$. Then $\Omega$ is said to be a $\mathbb{P}$-disproconnected subspace (or simply disproconnected- $\mathbb{P}$ ) if and only if either $\mathbb{P}(\underline{\Omega})=0$ or $\underline{\Omega}$ is disproconnected with respect to the normalized probability measure $\mathbb{P}\rceil_{\underline{\Omega}} / \mathbb{P}(\underline{\Omega})$ defined on $\left.\mathscr{F}\right\rceil_{\underline{\Omega}}$.

A subset of $\Omega$ is called a $\mathbb{P}$-proconnected subspace (or simply proconnected- $\mathbb{P}$ ) if and only if it is either a singleton or lies in $\mathscr{F}$ without being disproconnected- $\mathbb{P}$.

Since a singleton has exactly one element, there is no need to require a singleton to be measurable in Definition 2. Evidently, including the possibility of being a singleton in the definition of a proconnected subspace is merely a convenient normalization. Definition 2 implies that every nonmeasurable proconnected subspace is a singleton.

Although, due to the natural measurability constraint, the notion of a proconnected subspace seems less at liberty than that of a connected subspace, some useful fundamental results in the theory of connectedness carry over to proconnected subspaces. For instance, an arbitrary (measurable) union of proconnected subspaces with nonempty intersection is proconnected:

Theorem 4. Let $(\Omega, \mathscr{F}, \mathbb{P})$ be a probability space; let $\Theta$ be a nonempty set; let $\underline{\Omega}_{\theta} \subset \Omega$ be proconnected-P for all $\theta \in \Theta$; let $\underline{\Omega}:=\bigcup_{\theta \in \Theta} \underline{\Omega}_{\theta}$ lie in $\mathscr{F}$. If $\bigcap_{\theta \in \Theta} \underline{\Omega}_{\theta}$ is nonempty, then $\bigcup_{\theta \in \Theta} \underline{\Omega}_{\theta}$ is proconnected- $\mathbb{P}$.

Proof. Let $X: \underline{\Omega} \rightarrow\{0,1\}$ be measurable- $\mathscr{F}\rceil_{\underline{\Omega}}$ with the property that $\mathbb{P}_{X}(\{0\}) \mathbb{P}_{X}(\{1\})=$ 0. Then Theorem 3 implies that $X \underline{\Omega}_{\theta}$ is for all $\theta \in \Theta$ not a surjection. If $\omega_{0} \in \bigcap_{\theta} \underline{\Omega}_{\theta}$, then $X(\omega)=X\left(\omega_{0}\right)$ for all $\omega \in \underline{\Omega}$; and so $X$ is not a surjection. Thus $\underline{\Omega}$ is proconnected$\mathbb{P}$ by Theorem 3 .

Since proconnectedness is not intrinsic to measurability structures, it is anticipatable that a proconnected subspace in general need not have an apparent relationship with its closure or interior with respect to a given topology. However, we have

Proposition 2. Let $(\Omega, \mathscr{F}, \mathbb{P})$ be a probability space; let $A \in \mathscr{F}$ be proconnected-P; let $\underline{A} \supset A$ lie in $\mathscr{F}$. If $X^{1)}(\underline{A}) \subset X^{1)}(A)$ for all $\left.\mathscr{F}\right\rceil_{\underline{A}}$-measurable $X: \underline{A} \rightarrow\{0,1\}$, then $\underline{A}$ is proconnected- $\mathbb{P}$.

Proof. If $Y: A \rightarrow\{0,1\}$ is measurable- $\mathscr{F}\rceil_{A}$, then, since $A$ is by assumption proconnected$\mathbb{P}$, the function $Y$ is by Theorem 3 not a surjection. Let $X: \underline{A} \rightarrow\{0,1\}$ be measurable$\mathscr{F}\rceil_{\underline{A}}$. Then $X^{1)}(\underline{A}) \subset X^{1)}(A)$ by assumption. But $A \subset \underline{A}$ by assumption; so $X$ is not a surjection. 
For the restriction of a probability measure, we have

Proposition 3. Let $(\Omega, \mathscr{F}, \mathbb{P})$ be a probability space proconnected-P; let $\underline{\mathscr{F}} \subset \mathscr{F}$ be a sigma-algebra. Then $\Omega$ is proconnected- $\left.\mathbb{P}\right|_{\mathscr{F}}$.

Proof. If $\Omega$ is disproconnected- $\left.\mathbb{P}\right|_{\underline{\mathscr{F}}}$, then there is by Proposition 1 some nonempty $A \in \underline{\mathscr{F}}$ such that $A^{c}$ is nonempty and $\left.\mathbb{P}\right|_{\mathscr{F}}(A)=0$. But $\underline{\mathscr{F}} \subset \mathscr{F}$ by assumption; so $\Omega$ is disproconnected- $\mathbb{P}$.

The following result is an application of Theorem 1 ;

Theorem 5. Let $\Theta$ be a nonempty set; let $\Omega_{\theta}$ be a probability space with $\mathbb{P}_{\theta}$ denoting the given probability measure for all $\theta \in \Theta$. If the Cartesian product $\times_{\theta \in \Theta} \Omega_{\theta}$ is proconnected with respect to the product probability measure $\otimes_{\theta \in \Theta} \mathbb{P}_{\theta}$ defined on the cylinder sigmaalgebra of $\times_{\theta \in \Theta} \Omega_{\theta}$, then $\Omega_{\theta}$ is proconnected $-\mathbb{P}_{\theta}$ for all $\theta \in \Theta$.

Proof. The existence of the product probability measure on the cylinder sigma-algebra of the Cartesian product of arbitrary probability spaces is well-known.

The natural projection $\pi_{\theta}$ is measurable with respect to the cylinder sigma-algebra for all $\theta$; and we have $\Omega_{\theta}=\pi_{\theta}^{1)}\left(\times_{\theta} \Omega_{\theta}\right)$ for all $\theta$. Since

$$
\otimes_{\theta} \mathbb{P}_{\theta} \circ \pi_{\theta}^{-1)}=\mathbb{P}_{\theta}
$$

for all $\theta$, it follows by Theorem 1 that $\Omega_{\theta}$ is proconnected- $\mathbb{P}_{\theta}$ for all $\theta \in \Theta$.

We pause for now to list some miscellaneous results exploring proconnected and disproconnected spaces:

Proposition 4. Let $(\Omega, \mathscr{F}, \mathbb{P})$ be a probability space. (i) If $\Omega$ has cardinality $\geq 2$, and if $\mathbb{P}(\{\omega\})=0$ for some $\omega \in \Omega$, then $\Omega$ is disproconnected- $\mathbb{P}$. (ii) If $\Omega$ has cardinality $\geq 2$, then $\Omega$ is disproconnected with respect to every Dirac measure on $\mathscr{F}$. (iii) If there is some uncountable disjoint $\mathscr{A} \subset \mathscr{F}$ such that each element of $\mathscr{A}$ is nonempty, then $\Omega$ is disproconnected- $\mathbb{P}$. (iv) If $\mathbb{P}$ has some atom with cardinality $\geq 2$, and if this atom is a proper subset of $\Omega$, then $\Omega$ is disproconnected- $\mathbb{P}$.

Proof. The assertion (i) follows directly from Proposition 1 .

Let $\omega \in \Omega$; let $\mathbb{P}$ be the Dirac measure concentrated on $\{\omega\}$. Then $\mathbb{P}(\Omega \backslash\{\omega\})=0$, and Proposition 1 implies (ii).

Since $\mathscr{A} \subset \mathscr{F}$ is uncountable and disjoint, there are at most countably many $A \in \mathscr{A}$ such that $\mathbb{P}(A)>0$. Upon choosing some $\mathbb{P}$-null element of $\mathscr{A}$, which is necessarily nonempty by assumption, we obtain by Proposition 1 the assertion (iii).

To prove (iv), let $A$ be a proper subset of $\Omega$ that is an atom of $\mathbb{P}$ with cardinality $\geq 2$. Then $\mathbb{P}\left(A^{c}\right)=0$; and the conclusion follows from Proposition 1 . 
Immediate consequences are the following

Corollary 2. Let $\Omega$ be a probability space with $\mathbb{P}$ denoting the given probability measure; let $X: \Omega \rightarrow \mathbb{R}$ be measurable. If $\Omega$ is proconnected $\mathbb{P}$, then $\mathbb{P}_{X}$ is a Dirac measure concentrated at some point $x$ of $X^{1)}(\Omega)$ if and only if $X$ is constant on $\Omega$.

Proof. The "if" part is evident.

For the "only if" part, we observe that if $X$ is not constant on $\Omega$ then $X^{1)}(\Omega)$ has cardinality $\geq 2$. Since $\Omega$ is by assumption proconnected- $\mathbb{P}$, the set $X^{1)}(\Omega)$ is proconnected$\mathbb{P}_{X}$ by Theorem 1. But Proposition 4 implies that $X^{1)}(\Omega)$ is disproconnected- $\mathbb{P}_{X}$.

Proposition 5. Let $\Omega$ be a probability space with $\mathbb{P}$ denoting the given probability measure; let $\underline{\Omega}$ be a nonempty set. If $\Omega$ is uncountable and is proconnected- $\mathbb{P}$, and if $X: \Omega \rightarrow \underline{\Omega}$ is measurable, then $\mathbb{P}_{X}$ has no atom that is countable.

Proof. If $\mathbb{P}_{X}$ has some atom that is countable (necessarily nonempty), then, since its complement is by assumption nonempty, it follows by Proposition 4 that $\underline{\Omega}$ is disproconnected- $\mathbb{P}_{X}$. But $X$ is measurable by assumption, the space $\Omega$ is by Theorem 1 disproconnected- $\mathbb{P}$.

For a proconnected Hausdorff space equipped with a Borel probability measure, there is a natural restriction on its cardinality:

Theorem 6. Let $\Omega$ be a Hausdorff space; let $\mathbb{P}$ be a Borel probability measure over $\Omega$. If $\Omega$ is proconnected- $\mathbb{P}$, then $\Omega$ is countable.

Proof. If $\Omega$ is uncountable, then, since every singleton subset of $\Omega$ is closed and hence Borel-measurable, the space $\Omega$ is disproconnected- $\mathbb{P}$ by Proposition 4 .

In particular, we have

Corollary 3. If a Cartesian product $\Omega$ of Borel probability spaces is Hausdorff, and if $\Omega$ is proconnected with respect to the product probability measure defined on the Borel sigma-algebra of $\Omega$ generated by the product topology of $\Omega$, then every component space is countable.

On the other hand, disproconnectedness may be preserved under limiting operation:

Theorem 7. Let $\Omega$ be a metric space with cardinality $\geq 2$; let $\mathbb{P}_{n}$ be a Borel probability measure for all $n \in \mathbb{N}$; let there be some $\omega \in \Omega$ such that $\mathbb{P}_{n}(\{\omega\})=0$ for all $n \in \mathbb{N}$. If the densities $f_{n}$ of $\mathbb{P}_{n}$ converge to some density $f$ (Lebesgue-)almost everywhere, and if $\mathbb{P}: B \mapsto \int_{B} f$ on the Borel sigma-algebra of $\Omega$, then $\Omega$ is disproconnected- $\mathbb{P}$. 
Proof. Since $\left|f_{n}\right|_{L^{1}}=|f|_{L^{1}}=1$ for all $n$, the convergence assumption and the Riesz $L^{p_{-}}$ convergence theorem (see Novinger 2], a special case of which is known in probability theory as Scheffé's theorem; see Scheffé [3]) jointly imply that

$$
\sup _{B \text { Borel }}\left|\mathbb{P}_{n}(B)-\mathbb{P}(B)\right| \rightarrow 0 .
$$

But since $\{\omega\}$ is Borel-measurable, by the above convergence we have $\mathbb{P}(\{\omega\})=0$. That $\Omega$ is disproconnected- $\mathbb{P}$ then follows from Proposition 4

Theorem 8. Let $\Omega$ be a metric space; let $\mathbb{P}_{n}$ be a Borel probability measure over $\Omega$ for all $n \in \mathbb{N}$. If there is some nonempty closed $F \subset \Omega$ such that $F$ is a proper subset of $\Omega$ and $\left.\mathbb{P}_{n}\right\rceil_{F}=\mathbb{P}_{n}$ for all sufficiently large $n \in \mathbb{N}$, and if $\mathbb{P}_{n} \rightsquigarrow \mathbb{P}$ for some Borel probability measure $\mathbb{P}$ over $\Omega$, then $\Omega$ is disproconnected- $\mathbb{P}$.

Proof. Since $\mathbb{P}_{n}$ is by assumption concentrated on a fixed closed proper subset eventually, there is some nonempty open $G \subset \Omega$ such that $\mathbb{P}_{n}(G)=0$ for all sufficiently large $n$. But $\left(\mathbb{P}_{n}\right)$ converges to $\mathbb{P}$ weakly by assumption, by the fundamental portmanteau theorem in the theory of weak convergence we have

$$
\liminf _{n} \mathbb{P}_{n}(G) \geq \mathbb{P}(G)
$$

Since $\mathbb{P}(G)=0$, the desired conclusion follows from Proposition 4

A Euclidean space is disproconnected with respect to every "regular" nonatomic Borel probability measure:

Proposition 6. Let $k \in \mathbb{N}$; let $\mathbb{P}$ be a Borel probability measure over $\mathbb{R}^{k}$. If $\mathbb{P}$ is nonatomic, and if $\mathbb{P}(B)>0$ for some Borel $B \subset \mathbb{R}^{k}$, then $\mathbb{R}^{k}$ is disproconnected- $\mathbb{P}$.

Proof. Since there is some uncountable compact $K \subset B$ such that $\mathbb{P}(K)=0$, the space $\mathbb{R}^{k}$ is by Proposition 4 disproconnected- $\mathbb{P}$.

Before entering into the next major topic, we consider for a while the natural counterpart of the notion of topological hyperconnectedness:

Definition 3. Let $(\Omega, \mathscr{F}, \mathbb{P})$ be a probability space. Then $\Omega$ is said to be hyperproconnected- $\mathbb{P}$ if and only if for all nonempty disjoint $A_{1}, A_{2} \in \mathscr{F}$ it holds that $A_{1}$ is $\mathbb{P}$-dependent of $A_{2}$.

Thus hyperproconnectedness is not concerned with any partition of the underlying space. In general, there are many hyperproconnected spaces; for instance: 
Example 3. Let $\{0,1,2\}$ be taken as a discrete topological space; let $\mathbb{P}_{0}, \mathbb{P}_{1}, \mathbb{P}_{2}$ be, respectively, the Dirac measures concentrated at 0,1 , and 2 . Then $\Omega$ is hyperproconnected with respect to the probability measure $\mathbb{P}:=\frac{1}{3}\left(\mathbb{P}_{0}+\mathbb{P}_{1}+\mathbb{P}_{2}\right)$.

And certainly we have

Proposition 7. Every hyperproconnected probability space is proconnected.

The last main topic is the counterpart of the notion of connected components of a topological space:

Definition 4. Let $(\Omega, \mathscr{F}, \mathbb{P})$ be a probability space; let $\omega \in \Omega$. Then the set

$$
C_{\mathbb{P}}(\omega):=\bigcup\{A \in \mathscr{F} \mid A \ni \omega, A \text { is proconnected }-\mathbb{P}\}
$$

is called a $(\mathbb{P}-)$ proconnected component of $\Omega$ if and only if either $C_{\mathbb{P}}(\omega)$ is a singleton or $C_{\mathbb{P}}(\omega) \in \mathscr{F}$.

Then

Proposition 8. Let $(\Omega, \mathscr{F}, \mathbb{P})$ be a probability space; let $\omega \in \Omega$. Then $C_{\mathbb{P}}(\omega)$ is nonempty and is proconnected-P.

Proof. Since $\{\omega\}$ is by definition proconnected- $\mathbb{P}$, the set $C_{\mathbb{P}}(\omega)$ is nonempty.

The collection of all $\mathbb{P}$-proconnected elements of $\mathscr{F}$ that contain $\omega$ has nonempty intersection; so $C_{\mathbb{P}}(\omega)$ is proconnected- $\mathbb{P}$ by Theorem 4

Some of the basic maximality properties of connected components hold true for proconnected components by the same argument; we state them for concreteness:

Theorem 9. Let $\Omega$ be a probability space. Then (i) for every proconnected component of $\Omega$, there is no proconnected subspace of $\Omega$ that properly includes it, and (ii) the collection of all distinct proconnected components of $\Omega$ is a partition of $\Omega$.

Moreover, some nice properties of connected components are almost preserved by proconnected components:

Theorem 10. Let $\Omega$ a probability space; let $\underline{\Omega}$ be a measurable space. If $X: \Omega \rightarrow \underline{\Omega}$ is isomorphic, then for every $\mathbb{P}$-proconnected component $C \subset \Omega$ there is some $\mathbb{P}_{X}$ proconnected component $\underline{C} \subset \underline{\Omega}$ such that

$$
X^{1)}(C) \subset \underline{C} .
$$


Proof. Let $\omega \in \Omega$. Then Theorem 1 and the assumed isomorphicness of $X$ together imply that $X^{1)}\left(C_{\mathbb{P}}(\omega)\right)$ is proconnected- $\mathbb{P}_{X}$. If $\omega \in \Omega$, then, since $X(\omega) \in X^{1)}\left(C_{\mathbb{P}}(\omega)\right)$, we have

$$
X^{1)}\left(C_{\mathbb{P}}(\omega)\right) \subset C_{\mathbb{P}_{X}}(X(\omega)) .
$$

This completes the proof.

Theorem 11. Under the assumptions of Theorem 10 with the same notation, there is some bijection $f$ carrying $\mathbb{P}$-proconnected components to $\mathbb{P}_{X}$-proconnected components such that $C$ is isomorphic to $f^{1)}(C)$ via $X$ for all $\mathbb{P}$-proconnected components $C$.

Proof. By Theorem 10 we have

$$
X^{1)}\left(C_{\mathbb{P}}(\omega)\right) \subset C_{\mathbb{P}_{X}}(X(\omega))
$$

for all $\omega \in \Omega$; but $X$ being an isomorphism also implies

$$
C_{\mathbb{P}_{X}}(X(\omega)) \subset X^{1)}\left(C_{\mathbb{P}}(\omega)\right)
$$

for all $\omega \in \Omega$. The proof is completed by taking $f$ to be $X^{1)}$.

Given any nonempty set $\Omega$ and any $\omega \in \Omega$, denote by $\mathbb{D}^{\omega}$ the Dirac measure $A \mapsto$ $\mathbb{1}_{A}(\omega)$, defined on a sigma-algebra of $\Omega$, concentrated at $\omega$. The theory of proconnected components may be useful in checking isomorphicness:

Example 4. Let $\mathbb{P}:=\sum_{j \in \mathbb{N}} 2^{-j} \mathbb{D}^{(j, j)}$ on $2^{\mathbb{N}^{2}}$, being a probability measure concentrated on the diagonal of $\mathbb{N}^{2}$. Let $X: \mathbb{N}^{2} \rightarrow \mathbb{N}^{2}$ be measurable with

$$
\mathbb{P}_{X}=\sum_{j, k \in \mathbb{N}} 2^{-j-k} \mathbb{D}^{(j, k)}
$$

on $2^{\mathbb{N}^{2}}$. Then $C_{\mathbb{P}_{X}}(x)=\mathbb{N}^{2}$ for all $x \in \mathbb{N}^{2}$. But $j=k$ implies $C_{\mathbb{P}}(\{(j, k)\})=\{(n, n) \mid$ $n \in \mathbb{N}\}$, and $j \neq k$ implies $C_{\mathbb{P}}(\{(j, k)\})=\{(j, k)\}$ by Proposition 1 thus $X$ is not an isomorphism by Theorem 11 .

Example 5. Let $\mathbb{G}^{0,1}$ be the standard Gaussian distribution over $\mathbb{R}$; let

$$
\mathbb{P}:=\frac{1}{2} \sum_{j \in \mathbb{N}} 2^{-j} \mathbb{D}^{j}+\frac{1}{2} \mathbb{G}^{0,1}
$$

on the Borel sigma-algebra of $\mathbb{R}$. Given an uncountable measurable space $\underline{\Omega}$ equipped with the countable-cocountable sigma-algebra, consider a measurable $X: \mathbb{R} \rightarrow \underline{\Omega}$. Suppose $\mathbb{P}_{X}(A)=1$ if $A^{c}$ is countable, and is $=0$ if $A$ is countable. 
Then a $\mathbb{P}_{X}$-proconnected component of $\underline{\Omega}$ is by Proposition 1 precisely a singleton subset of $\underline{\Omega}$. However, we have $C_{\mathbb{P}}(n)=\mathbb{N}$ for all $n \in \mathbb{N}$, which is certainly not in bijection with any $\mathbb{P}_{X}$-proconnected component of $\underline{\Omega}$. It follows by Theorem 11 that $\mathbb{R}$ is not isomorphic to $\underline{\Omega}$ via $X$.

An automorphism of a proconnected probability space can only induce probability measures of a certain kind:

Theorem 12. Let $(\Omega, \mathscr{F}, \mathbb{P})$ be a probability space proconnected- $\mathbb{P}$. If $X$ is an automorphism of $\Omega$, then $\mathbb{P}$ is not singular with respect to $\mathbb{P}_{X}$.

Proof. The conclusion is evident if $\Omega$ is a singleton.

Suppose $\Omega$ has cardinality $\geq 2$. There is by assumption some $A \in \mathscr{F}$ such that $\mathbb{P}(A)=1$ and $\mathbb{P}_{X}(A)=0$. Now, since $A$ is nonempty, the $\mathbb{P}_{X}$-proconnected component of every point of $A$ is by Proposition 1 a singleton; but the only $\mathbb{P}$-proconnected component is $\Omega$. The desired conclusion then follows from Theorem 11

\section{References}

[1] Federer, H. (1996). Geometric Measure Theory, reprint of the first edition. Springer.

[2] Novinger, W. P. (1972). Mean convergence in $L^{p}$ spaces. Proc. Amer. Math. Soc. 34 $627-628$.

[3] Scheffé, H. (1947). A useful convergence theorem for probability distributions. Ann. Math. Stat. 18 434-438. 